\title{
Aided and unaided decisions with imprecise probabilities in the domain of losses
}

\author{
David V. Budescu • Stephen B. Broomell • \\ Robert J. Lempert • Klaus Keller
}

Received: 29 September 2012/ Accepted: 20 December 2013/Published online: 15 January 2014 (C) Springer-Verlag Berlin Heidelberg and EURO - The Association of European Operational Research Societies 2014

\begin{abstract}
We report results of a series of experiments on decision-making in the presence of irreducibly imprecise probabilities of negative and undesirable outcomes. Subjects faced decisions among actions where the payoffs depend on the probability of drawing balls from an urn whose composition was not fully known. Consistent with the vagueness avoidance hypothesis, Decision Makers (DMs) displayed systematic preferences for safe actions even at a high premium. This tendency grew with increased vagueness, characterized by the width of the interval of plausible probabilities. We compared two decision aids that portray these imprecise probabilities in different ways: one aid calculates the expected value of alternative actions contingent on probability distributions provided by the DMs, and the other displays graphically the distribution of the conditional expected value of the actions over the entire range of plausible probabilities. Access to either decision aid reduced vagueness avoidance and the type of aid used systematically influenced the way DMs approached the problem. We compared the DMs' choices with predictions of decision models for decision under ignorance and under risk. We found support for
\end{abstract}

Electronic supplementary material The online version of this article (doi:10.1007/s40070-013-0023-4) contains supplementary material, which is available to authorized users.

\footnotetext{
D. V. Budescu $(\bowtie)$

Department of Psychology, Fordham University, Bronx, NY 10458, USA

e-mail: budescu@fordham.edu
}

\section{S. B. Broomell}

Department of Social and Decision Sciences, Carnegie Mellon University, Pittsburgh, PA, USA

\section{R. J. Lempert}

RAND Corporation, Santa Monica, CA, USA

\section{K. Keller}

Department of Geosciences, and Earth and Environmental Systems Institute,

The Pennsylvania State University, State College, PA, USA 
the conservative maxi-min criterion, but a subjective expected value model with probabilities inferred from the partial information available also performed well, especially for low levels of vagueness and in the presence of decision aids. These findings suggest some initial implications for the debate over how to best characterize imprecise probabilistic information for policy-makers when decisions involve irreducible uncertainties, such as climate change.

Keywords Ambiguity · Deep uncertainty · Decision aids · Imprecise probabilities · Maxi-min - Subjective expected value $\cdot$ Vague probabilities

Mathematics Subject Classification 91B06 - 91B08 -91B16 -91E99

\section{Introduction}

The presentation of probabilities and other information about risks can have an important influence on an individual's choices (e.g., Fiedler and Unkelbach 2011; Lipkus 2007; Sanfey and Hastie 1998). Decision Makers (DMs for short) face vague or imprecise probabilities ${ }^{1}$ in many real-world decisions. In cases where one cannot specify a proper probability distribution over the possible outcomes, as required for a traditional decision analysis guided by utility theory, uncertainties can be communicated as approximate probabilities, (e.g., "around 0.30"), numerical ranges (e.g., "between 0.10 and 0.50 ", or "not more than 0.40 "), or verbal descriptions (e.g., "highly unlikely"). The effects of various presentation modes of such vague information have not been studied systematically. We report results of three experiments designed to test how the treatment of vagueness in decision aids affects individuals' choices. We use a simple experimental setup where subjects face a stylized decision where the information about key probabilities is imprecise and compare the effects of two decision aids, one that stresses the best guess integrated across all probabilities and the other which stresses the irreducible imprecision of the probabilities.

This study is motivated in part by a desire to inform the debate over how to best characterize and present imprecise probabilistic information for policy-makers. Climate change presents an archetypal example. While the basic physical mechanisms of anthropogenic climate change are well understood (Arrhenius 1896), the specific magnitude and potential future impacts are not (e.g., Keller et al. 2008; Morgan and Keith 1995; Welsch 1995). Estimates of the probability density functions for key parameters describing both the climate and energy economic systems that affect it vary considerably across experts (e.g., Morgan and Keith 1995; Zickfeld et al. 2007). Thus, scientists and economists can provide policy makers only vague information regarding the likelihood of serious climate change impacts or the likelihood that various response options will prove cost-effective solutions. Another feature of many decision problems in the climate and environmental context is that they are driven by undesirable outcomes (e.g., minimize effects of climate change or pollutants). Consequently, most of our discussion and our experiments will focus on negative outcomes (losses).

\footnotetext{
${ }^{1}$ We use the terms vague and imprecise probability interchangeably throughout the text.
} 
The presence of such irreducible forecasts regarding climate change decisions has spawned extensive debates about how to best communicate this information. Some embrace the standard Bayesian view and argue that regardless of any imprecision, experts ought to provide their best-guess estimates of the probabilities because government officials require such information to properly assess alternative policy options (Giles 2002; Moss and Schneider 2000; Webster 2003). Others argue that information about vagueness is crucial to the policy debate and that experts should describe how various policy options perform over a wide range of deep uncertainties $^{2}$ (Casman et al. 1999; Lempert et al. 2004).

\subsection{Effects of vagueness on decisions}

Ellsberg (1961) gets credit for highlighting the impact of (im)precision in probabilities on decision making. The "Ellsberg paradox" shows that most people prefer making decisions based on precise probabilities even at a premium. This vagueness aversion $^{3}$ has been demonstrated in numerous laboratory experiments and generalized to health, environmental, and other contexts (see Becker and Brownson 1964; Camerer and Weber 1992; Curley and Yates 1985; Du and Budescu 2005; Fox and Tversky 1995; Hogarth and Kunreuther 1989; Keren and Gerritsen, 1999; Kramer and Budescu 2005; Kuhn and Budescu 1996; for partial reviews and illustrative examples).

Much of the literature seeks to establish the boundaries of this empirical regularity and explain its psychological sources (e.g., Curley et al. 1986). Most of the work has focused on situations where all the components of the decision, except the precision of the probabilities, are constant to isolate the net effect of the vagueness. In fact, one prominent explanation for the observed behavior, the comparative ignorance hypothesis (Fox and Tversky 1995; Fox and Weber 2002), suggests that the results are driven by the salience of the direct contrast between precise and vague states. Specifically, it predicts vagueness aversion in comparative settings, such as pair-wise choices between precise and imprecise options, but anticipates smaller (or no) effects in the absence of such explicit comparisons, (e.g., pricing, rating, or determining cash equivalents of options, presented in isolation). $\mathrm{Du}$ and Budescu (2005) presented experimental results consistent with this prediction (see also, Trautmann et al. 2011).

As Camerer and Weber (1992) pointed out in their often-cited review, vagueness aversion is not universal. Recent papers have documented the differential distributions of attitudes to vagueness for various elicitation methods (e.g., Du

\footnotetext{
${ }^{2}$ Deep uncertainty has been defined (Lempert et al. 2003) as the condition in which DMs do not know, or do not agree upon (1) the appropriate models that relate actions to consequences, (2) the probability distributions over key input parameters to those models, and (3) how to value the desirability of alternative outcomes. The term thus includes the condition of imprecise or vague probabilities.

3 Ellsberg used the term ambiguity to refer to imprecisely specified probabilities. Budescu et al. (1988) have argued against this convention and advocated using vagueness or imprecision in this context (see also Casey and Scholz 1991). According to Budescu and Wallsten's (1995) taxonomy, an event is ambiguous if its description is subject to multiple interpretations (e.g., "in the fall of 2008, the stock market will increase moderately"), and a probability distribution is vague if the DM cannot associate all events in the sample space with unique and precise numerical probabilities.
} 
and Budescu 2005 for choices and CEs; Trautmann et al. 2011 for choices and WTP). Several papers have shown that attitudes to vagueness are typically only secondary considerations in decisions. They are not as important as criteria such as attitudes towards risk or magnitude of outcomes and are, therefore, malleable (Du and Budescu 2005; Kuhn and Budescu 1996; Kuhn et al. 1999; Onay et al. 2012).

Nevertheless, aversion to imprecision is prevalent and it is indeed the modal pattern in choices, (Kramer and Budescu 2005; Trautmann et al. 2011) CEs, (Budescu et al. 2002) and WTP (Trautmann et al. 2011). This aversion is also invoked routinely to explain certain empirical regularities in applied settings (e.g., Chen and Epstein 2002 for finance, and Kunreuther et al. 1995, for insurance). We refer to this general tendency as the vagueness aversion hypothesis and we also use it to derive our global-group level-hypotheses.

\subsection{Effects of presentation of probabilistic information on decisions}

It is widely recognized that judgments of uncertainty (and subsequent decisions that rely on them) are sensitive to the form, format, and framing of the presentation of the sample space and the governing stochastic process. For example, Gigerenzer et al. (1994) have documented differences between decisions based on probabilities and on natural frequencies, and argue for the superiority of the latter. In a compelling illustration Hoffrage et al. (2000) showed that the rate of correct solution of (Bayesian) diagnostic problems by medical professionals increased dramatically when the problems were presented in terms of natural frequencies. Budescu and Fischer (2001) asked subjects to make pair-wise choices between gambles that were equivalent, but involved different implementations of the chance apparatus (e.g., choose between: Gamble A: win \$X if a fair coin lands on "Heads", or Gamble B: win \$X if a "Red" card is chosen from a complete deck of cards). Their subjects displayed systematic preferences for presentations involving larger sample spaces (e.g., 52 possible cards versus 2 possible sides of a coin), and fewer stages that allow for quick resolution of uncertainty (e.g., Gamble A: win \$X if a fair coin lands on "Heads", over Gamble B: win \$X if a coin lands on the same side-either "Heads" or "Tails"—-twice in a row). MacGregor and Slovic (1986) and Sanfey and Hastie (1998) performed experiments in which information about runners was presented in various ways to subjects who had to predict their time on a future marathon race. Both studies found that presentation format affected subsequent predictions. These studies suggest that one can think of various presentation formats as "decision aids".

There is a large and highly dispersed literature on decision aids and a comprehensive review is beyond the scope of this paper. Instead, we focus on some key results that are relevant to our work. The first is, simply, that having access to decision aids affects both the choices people make and their attitudes about their decisions. Hung et al. (2007) showed that participants who could use a decision support system in an investment context performed better and were more confident than subjects in a Control group who received the same amount of information but did not have decision aids. Various presentation modes can improve decisions, as 
shown by Keller (1985a, b) who documented reduced rates of violations of certain tenets of rationality through various graphical representations.

Kleinmuntz and Schkade (1993) claim that various presentation modes can facilitate certain cognitive decision processes and hinder others. MacGregor and Slovic (1986) and Sanfey and Hastie (1998) suggest that the key drivers in this process are the salience/prominence of the information and the similarity/ compatibility between the nature of the data and the displays. Thus, different decision aids can increase the likelihood that DMs who respond to these cues would choose differently (see Ibrekk and Morgan 1987; Johnson et al. 1988; Todd and Benbasat 1994; for some empirical demonstrations). Conversely, poorly designed aids can mislead DMs into inferior decisions (e.g., Arunachalam et al. 2002).

\subsection{The impact of different treatments of vague probabilities on decisions}

We combine these two streams of literature by studying how different presentations of vague uncertainties affect decisions. To achieve this goal we developed and implemented a new design for the study of vague probabilities. Most previous work has focused on the effect of different sources of uncertainty (Abdellaoui et al. 2011) on decisions. Inspired by Ellsberg's seminal paper people have studied how DMs choose between, or are willing to pay for, options that involve different source functions (i.e., various levels of precision) but are otherwise identical. Thus the key contrast in this work is the presence and, occasionally, the degree of imprecision. In our study we have a single common source function, and study how people evaluate, and choose among, several actions that are available to them in the presence of (identical) irreducible vague probabilities. The key issue is how DMs incorporate a given level of vagueness in the decision environment into their decision process. Another way in which our study differs from most previous work is that we invoke a more complex probabilistic model based on repeated samples from a certain data generating process (see more details below) to represent uncertainty. Both changes contribute to making this a more realistic scenario that mimics the types of decisions people encounter in such cases.

Consider a target event, $E$, with probability, $P$. The target probability is unknown to the DM but it can be narrowed to an interval with two sharp bounds, $P_{\mathrm{L}}$ and $P_{\mathrm{U}}$, such that $0 \leq P_{\mathrm{L}}<P<P_{\mathrm{U}} \leq 1$. The DM needs to choose one of $m$ possible actions $A_{1}, A_{2} \ldots, A_{i}, \ldots A_{m}$ that lead to various outcomes contingent on the realization of the target event, $E$. In our experiments we Control the vagueness of the target probability by manipulating $P_{\mathrm{L}}$ and $P_{\mathrm{U}}$, and the outcomes associated with the various actions, $A_{i},(i=1 \ldots m)$. The impact of imprecision declines as (a) the range of probabilities is reduced, so the decision resembles more a typical decision under risk with a precise $P$, and/or (b) the variance of the payoffs for each possible action is reduced, thus making the uncertainty less relevant. A "safe" action that offers a fixed payoff, regardless of $P$ and its limits $\left(P_{\mathrm{L}}\right.$ and $\left.P_{\mathrm{U}}\right)$, eliminates the effects of the underlying imprecision.

We use two decision aids. Both present to the judges expected values of the various actions but rely on two distinct approaches to dealing with vagueness. One seeks to minimize (if not totally eliminate) the inherent vagueness through 
integration over the domain of interest, and the other seeks to highlight its effects by displaying its impacts. The first method provides a single summary in the form of the expected value of each specific action integrated across the range of probabilities. The second method provides a display of the expected value of each specific action as a function of target probability, across the full range of probabilities. The first aid is an expected value calculator that allows DMs to specify hypothetical probability distributions over the $\left[P_{\mathrm{L}}, P_{\mathrm{U}}\right]$ range, say $\Pi_{j}$, and uses this distribution to calculate the expectations of each action, $E\left(A_{\mathrm{i}} \mid \Pi_{j}\right)$ $(i=1 \ldots m)$. This aid mimics the approach taken by most decision analytic studies of climate change (e.g., Nordhaus 1994; Kann and Weyant 2000). It provides an effective way of eliminating imprecision by combining it, subject to the assumptions encapsulated in $\Pi_{j}$, with the outcomes, and allows the DM to rank order the actions, $A_{i}$. We refer to this aid as Summary. The second decision aid takes a diametrically opposite approach: instead of eliminating the imprecision, it highlights it and explicitly displays its consequences. This aid, which we labeled Display, shows the expected outcome of each action for all probabilities in the relevant range. More specifically, it plots $\operatorname{EV}\left(A_{i} \mid P\right)$ for all $P_{\mathrm{L}}<P<P_{\mathrm{U}}$. It mimics decision analytic approaches that attempt to identify climate policy as robust against vague probabilities (Borsuk and Tomassini 2005; Brock and Xepapadeas 2003; Hall et al. 2012; Lempert et al. 1996, 2003; McInerney et al. 2012), as it helps DMs to identify actions that dominate, or are dominated by, others and to form impressions about relative advantages of the various actions, i.e., the subset of the $\left[P_{\mathrm{L}}, P_{\mathrm{U}}\right]$ range where $A_{i}$ is the best, worst, etc.

Our experiments were designed to test directly four hypotheses. In addition, we use the data collected in the various studies to test a fifth hypothesis about the nature of the decisions models used by the DMs. Based on the vagueness aversion hypothesis, we hypothesize:

H1: DMs will display systematic preferences for the "safest" action. We predict that this tendency will increase as a function of (a) the degree of vagueness and (b) the overall probability of the undesirable outcome (as operationalized by the midrange of the feasible probabilities).

We hypothesize that the attitude to vagueness (and the pattern of revealed preferences) will be sensitive to the mode of presentation underlying the various decision aids. In particular,

H2: Access to the decision aids will reduce the level of choice of the safe action, and will favor more risky actions.

A more specific prediction relates the nature of the decision aid to the principle used to make the eventual choice. Consistent with literature on input-output compatibility (e.g., Shafir 1995) we predict,

H3: Access to the various decision aids will trigger different cognitive processes and can cause people to favor different actions. We predict that the Summary aid will cause people to focus on the integrated mean value and induce preferences for actions with higher expected values (under the 
probability distributions studied). On the other hand, exposure to the Display aid will cause DMs to favor the actions that are perceived as superior for a large portion of the relevant probability range.

Salvadori et al. (2001) define subjective confidence as the strength of belief in the goodness, accuracy, and appropriateness of one's judgments and/or decisions and, as such, it is an indicator of one's commitment to and willingness to act upon them (e.g., Budescu and Rantilla 2000). This leads to,

H4: Confidence in one's decisions will be higher (lower) in more precise (vaguer) cases, and access to the decisions aids will boost the DMs' level of confidence in their decisions.

Probably the most interesting problem from a behavioral decision theoretical point of view is what decision process is adopted by the DMs facing these decisions. This is a particularly intriguing question since the standard taxonomy of decision models (e.g., French 1986; Luce and Raiffa 1954) distinguishes between, and offers multiple models for, decisions under ignorance (probabilities unknown) and decisions under risk (probabilities known or estimable), but there are no prescriptions for decisions under vagueness with partially known probabilities. In fact, there is no uniformly accepted normative solution to the problem of decision with imprecise probabilities (e.g., Etner et al. 2012; Manski 2010; Troffaes 2007). Recently, this question was raised in the context of climate change (see Kunreuther et al. 2013). Our experiments provide a unique opportunity to determine how DMs perceive such problems and test which class of models best fit decisions in this grey area. We hypothesize,

H5: In the presence of irreducible vagueness decisions will reveal interindividual differences, with some people acting in line with models of decisions under ignorance (ignoring probabilities) and others imputing estimates of the probabilities and acting in line with a Subjective Expected Value (SEV) model. As the level of vagueness increases, the rate of reliance on the SEV will decrease.

\subsection{The experimental task}

The following task that was designed to test these hypotheses about the effects of aids on decisions with imprecise probabilities was used in three experiments, subject to appropriate changes in its parameters. Although our design is motivated by decisions in the context of climate change we test these two prototypical decision aids in a context-free setting to reduce the impact of potential biases related to beliefs and ideological stances with regard to climate change.

The DM is presented with an urn with 100 balls. A fraction of these balls, $P(0<P<1)$, is red; the rest are white. The DM does not know the exact value of $P$, but knows its lower and upper bounds ( $P_{\mathrm{L}}$ and $P_{\mathrm{U}}$, respectively). The DM is endowed with $\$ e$ and has to choose between the following actions: 
- Action $A_{1}$ : The DM draws two balls with replacement. If at least one ball is white the DM keeps the $\$ e$; if both are red the DM surrenders $\$ e$ and is left with 0 . $^{4}$

- Action $A_{2}$ : The DM surrenders $\$ f$ (where $0<f<e$ ) and keeps the remaining $\$(e-f)$. No draws from the urn take place.

- Action $A_{3}$ : The DM surrenders a small amount $\$ y(0<y<f<e)$ and draws two balls with replacement. If at least one ball is white the DM keeps the remaining $\$(e-y)$. If both balls are red the DM can draw a third ball from the urn. If it is white the DM keeps the remaining $\$(e-y)$. If the third ball is also red, the DM loses another $\$ z(z<(e-y))$ and keeps the remaining $\$(e-y-z)$.

Figure 1 presents the decision trees corresponding to the three actions. For any fixed $P$ in the $\left(P_{\mathrm{L}}, P_{\mathrm{U}}\right)$ range the expected value of these actions is given by: $\operatorname{EV}\left(A_{1}\right)=\$ e\left(1-P^{2}\right) ; \operatorname{EV}\left(A_{2}\right)=\$(e-f) ;$ and $\operatorname{EV}\left(A_{3}\right)=\$(e-y)-z P^{3}$. Note that the Figure and its parameterization reflect the objective structure of the problem and its normative analysis, and not its subjective representation (which may involve non-linear transformations to the values) by the various DMs.

The game was designed such that the expected payoffs for the uncertain actions $\left(A_{1}\right.$ and $\left.A_{3}\right)$ are non-linear in the (imprecise) probability of choosing a red ball, $P$, to accentuate any sensitivity to potentially large and poorly characterized risks. In this sense the game's design represents a simplified and abstract instantiation of the abrupt climate change policy problem. Action $A_{1}$ corresponds to a "Business as Usual" strategy that imposes no costs for reducing greenhouse gas emissions but risks substantial adverse impacts from climate change. If one knew $P$, this would be a typical risky choice. The safe action, $A_{2}$, corresponds to an aggressive reduction of emissions that would greatly reduce the risk of such adverse impacts. Action $A_{3}$ represents an intermediate alternative with reduced risk through an action that resembles purchasing insurance. Also, consistent with any analysis of the climate change problem the outcomes are negative, i.e., departures from the status quo in the undesirable direction.

\section{Experiment 1: access to decision aids affects decisions}

This first experiment compares choices among the three actions either with one of the decision aids (Summary, Display) or without a decision aid.

\subsection{Method}

\subsubsection{Participants}

Eighty students at the University of Illinois at Urbana-Champaign (35 men, 43 women, 2 information missing) were recruited by posting advertisements on campus

\footnotetext{
${ }^{4}$ Some researchers let the subjects select the winning/losing color in the urns. Given the context and the association of Red with potentially undesirable and dangerous outcomes we decided to make red the losing color for all participants. This choice should have no impact on our experimental manipulations.
} 
Fig. 1 Decision trees for the three actions available to the DMs

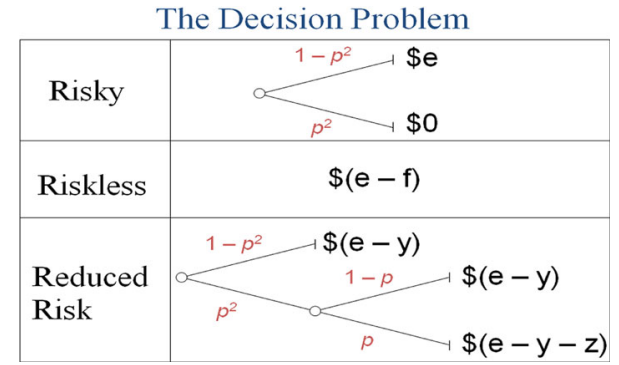

and electronic bulletin boards regarding a decision experiment offering the possibility of earning cash prizes.

\subsubsection{Design and procedure}

Participants were randomly assigned to one of six groups defined by crossing 3 decision aids with the possibility of purchasing information. The three decision aids are labeled Display, Summary and Control. In the Control group participants did not have access to any decision aid. In the Display group subjects saw a graphical display of the expected payoffs for each action across the entire range of probabilities for drawing a red ball. The graph displayed curves of the computed EV for all actions across all the relevant values of $P$. If subjects purchased information, only the restricted range was displayed (see Figure 2). In the Summary condition the subjects saw an interactive calculator that computed the expected values for each action. It allowed participants to input a distribution over the vague probability range to place more (or less) weight on different probabilities in the range. The default was a uniform distribution over $\left[P_{\mathrm{L}}, P_{\mathrm{U}}\right]$, but subjects could specify as many as three additional distributions over the range (or the narrower range, if information was purchased), and the program re-calculated the expected value for each distribution (see Fig. 3). The relevant probability range was divided into intervals of length 0.1 (e.g., $0-0.1,0.1-0.2$, etc.). The second factor was the option of purchasing information to reduce the range of possible values of $P$ before choosing an action. For a fixed amount $\$ c<\$ e$ the DM could reduce the upper bound, $P_{\mathrm{U}}$, by 0.20. This information purchase reflects the option of investing in research to reduce the imprecision associated with the likelihood of climate change.

Participants were run individually in a laboratory. The experimental materials were composed of (a) 18 opaque urns that represented independent choices, (b) two clear bins that that held large quantities of white and red balls, and (c) a computer. The 18 opaque urns were prepared prior to the participant's arrival to the lab by partially filling each urn with balls in a composition unknown (and not visible) to the participants.

Each participant was provided with instructions and a consent form. Participants made 18 independent sets of decisions (one for each of the opaque urns). For each one, the experimenter described to the participant the parameters of the urn and the actions using neutral labels $(\mathrm{A}, \mathrm{B}, \mathrm{C})$ to avoid any connotations associated with 


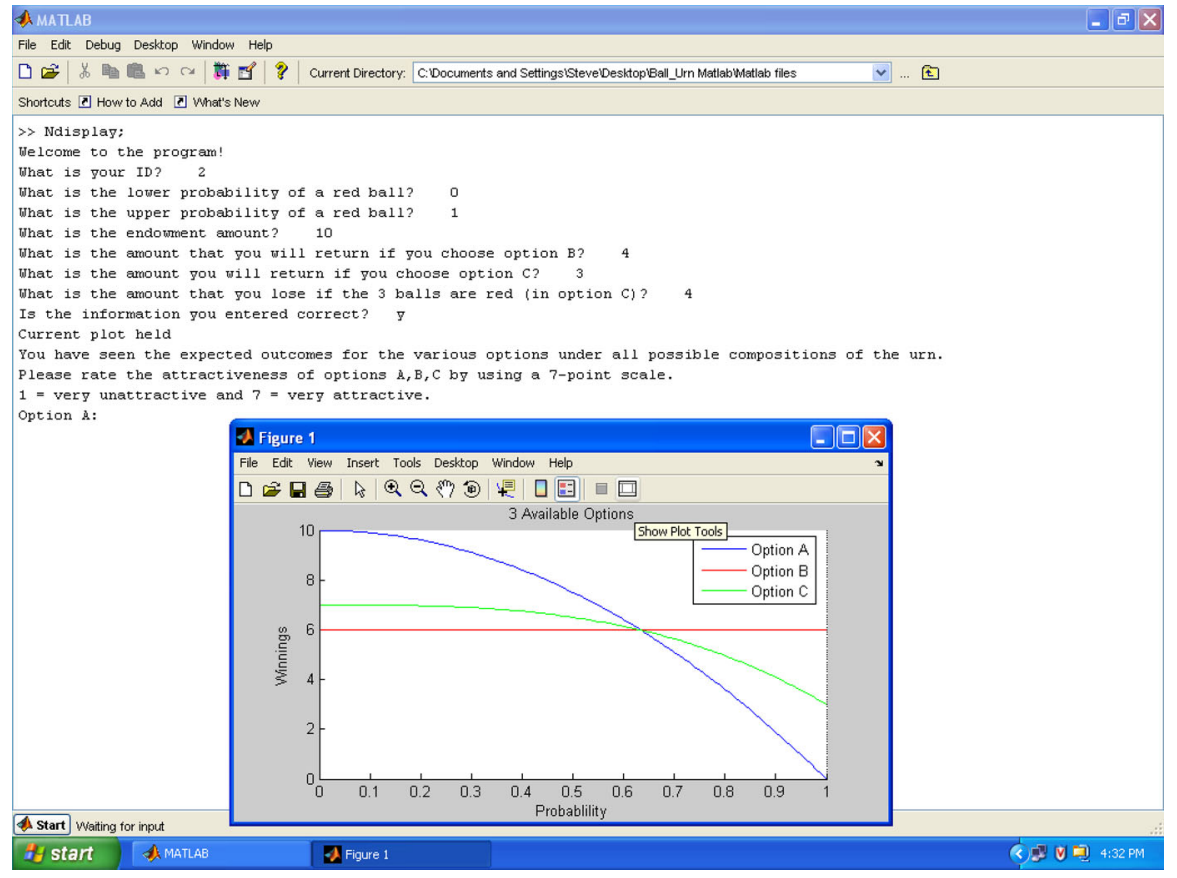

Fig. 2 Screen for the display condition

value laden terms (risk, insurance, uncertainty, etc.). Depending on condition, if the subject decided to purchase information the experimenter took 20 white balls from the urn, showed them to the subject, and replaced them in the urn prior to adding the extra balls. ${ }^{5}$ Participants were then instructed to fill the urn by adding white and red balls from the two transparent bins so that there was a total of 100 balls in the urn. In all conditions participants knew how many balls they added so that they could infer the bounds of the probability of drawing a red ball.

For example, to implement the case where $P$ is between 0.3 and 0.7 , the subjects were told that there were already 40 red and white balls in the urn and the outcomes associated with the various actions. The participant then counted and added 30 white and 30 red balls so that the urn had exactly 100 balls. Participants who did not purchase information knew that the number of red balls was at least 30, and at most 70. Information purchase allowed the participant to infer that the number of red balls in the urn was at least 30 and at most 50 after seeing that there were at least 20 white marbles in the urn.

Next, the participant and the experimenter turned to the computer and the participant keyed in all the parameters $\left(e, f, c, y, z, P_{\mathrm{L}}, P_{\mathrm{U}}\right)$. In our example,

\footnotetext{
5 Participants were told that they could see up to 20 white marbles from the urn, and if there were less than 20, the experimenter would pull out all the white marbles. Participants were not aware that there would be at least 20 white marbles in each urn.
} 


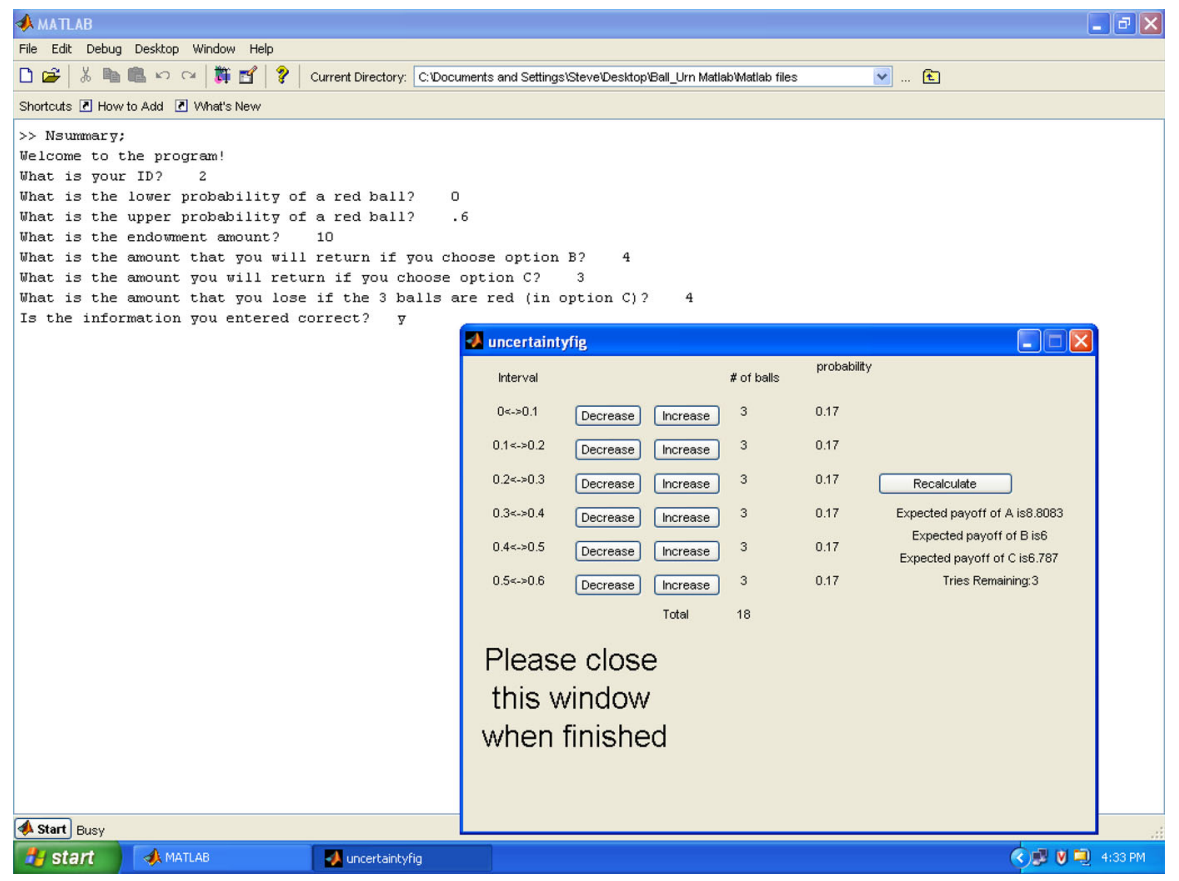

Fig. 3 Screen for the summary condition

participants would enter $e, f, c, y, z$ and $P_{\mathrm{L}}($ red $)=0.30$ and $P_{\mathrm{U}}$ (red) $=0.70$ (those who purchased information keyed in $P_{\mathrm{L}}(\mathrm{red})=0.30$ and $P_{\mathrm{U}}($ red $\left.)=0.50\right)$. Each parameter was double checked by experimenter to ensure that the participant understood the problem scenario.

Finally, the subjects consulted their aids (depending on condition), rated the attractiveness of each action on a 1-7 scale, selected one of the actions, and rated their confidence in the choice (using a 1-7 scale ranging from Not at all confident to Extremely confident).

We fixed three parameters across all 18 urns: the endowment $e$ was set at $\$ 10$; the voluntary fine for the safe action $f$ was set at $\$ 4$; and the cost of vagueness reduction information was fixed at $c=\$ 2$. We manipulated the parameters of action $A_{3}$, the cost of "insurance" $y$ and the additional loss $z$, using two pairs of values with a constant sum: $(y=\$ 2, z=\$ 5)$ and $(y=\$ 3, z=\$ 4)$. Table A1 in the Appendix shows the nine ranges of vague probabilities. The table's entries show the probability's lower and upper bounds and display the nine cases as a function of the range and midrange of the intervals they define.

To recapitulate, we ran six groups of subjects defined by crossing (a) the possibility of purchasing information and (b) one of three decision aids. Each subject judged 18 urns defined by crossing: (a) 9 levels of vagueness and (b) two 
pairs of $(y, z)$. The dependent variables of interested were ratings of each action, the action chosen $\left(A_{1}, A_{2}\right.$ or $\left.A_{3}\right)$, and confidence in one's choice.

One of two urns with the highest level of imprecision was used on the first trial (see Appendix). After this first decision was made, if necessary (no balls were selected when the safe action, $A_{2}$, was selected), the subject picked two balls with replacement from the urn (if necessary, they picked a third one when the reduced risk action, $A_{3}$, was selected) and the earnings were determined and recorded. After the first choice was completed, the process (with the exception of the actual draws from the urn) was repeated for each of the remaining 17 urns and the experimenter recorded the subjects' ratings and choices. The order of presentation varied as participants were allowed to choose the next urn.

Participants were paid based on their outcome of 3 of the 18 urns: Trial one and two additional ones that were randomly chosen and played according to the participant's recorded choice at the end of the session. The experiment lasted about one hour and subjects earned between $\$ 8$ and $\$ 30$. The mean payoff was $\$ 21$ $($ median $=\$ 20, \mathrm{SD}=\$ 5.3)$.

\subsection{Results}

Various sections of our analysis use different dependent variables. The main analysis relies on the normalized ratings associated with each of the three actions, but in some cases we give up the detailed information of these ratings and simply focus on the action chosen by the DM $\left(A_{1}, A_{2}\right.$ or $\left.A_{3}\right)$. The test of $H 4$ is based on the DM's reported confidence levels.

Half the subjects were offered the possibility to purchase information to reduce the vagueness, but they took advantage of this option on only $18 \%$ of the cases (24\% in the Control group, $17 \%$ in the Display, and $15 \%$ in the Summary group). DMs purchased information more frequently in urns with wider ranges of probabilities. Given the low rate of information purchase we will not use this factor in the analysis. In cases where vagueness reducing information was purchased the choices were analyzed according to the reduced vagueness and midrange. For example, if the probability in the original urn was between 0.3 and 0.7 , and the subject purchased information, the choice was analyzed as having a probability between 0.3 and 0.5 and a midrange of $0.4 .^{6}$

\subsubsection{Analysis of normalized ratings}

The normalized ratings are obtained by dividing the raw rating (1-7) by the sum of all the ratings for a given decision:

Normalized rating $\left(A_{\mathrm{i}}\right)=100 \times\left[\operatorname{Raw} \operatorname{rating}\left(A_{\mathrm{i}}\right) / \Sigma \operatorname{Raw} \operatorname{rating}\left(A_{\mathrm{i}}\right)\right]$,

\footnotetext{
${ }^{6}$ Information purchase was more desirable under conditions where there was a wider range of probabilities. No participant purchased information for decision problems with the narrowest range of probabilities $=0.4$. This means that the lowest midrange is 0.3 and the narrowest range is 0.4 for all analyses regardless of information purchase.
} 
so they are bounded by 0 and 100 . The normalization eliminates differences between the overall attractiveness of the various decision problems. ${ }^{7}$ The normalized ratings of the three actions were analyzed by a 4-way MANOVA with one between-subjects factor (the decision aid), and three within-subjects factors (range of the probability interval, midrange of the probability interval, and the fines associated with action $\mathrm{A}_{3}$ ). The mean normalized ratings of $A_{1}, A_{2}$, and $A_{3}$ are presented in Table 1, as a function of each of these factors.

Overall, the DMs in the Control group rated the safe action $\left(A_{2}\right)$ as the most attractive and action $A_{1}$ as the least attractive, but the rankings of the actions were reversed with decision aids. Indeed, we found significant differences between the three decision aids $\left(F_{(4,152)}=4.27 ; P<0.05\right){ }^{8}$ Post-hoc tests (Tukey HSD) confirmed that both decision aids generated ratings that differed significantly from the Control, but the difference between Summary and Display was not significant. The mean ratings documented several clear empirical regularities in response to the parameters of the decisions: as the range and midrange of the probability increase (making the situation vaguer and less desirable) the safe action $\left(A_{2}\right)$ was rated higher in all groups. On the other hand, as the range and midrange of the probability interval decrease (making the situation more precise and less aversive) the Action $A_{1}$ became more attractive in all groups. These effects of the range and midrange were additive. Indeed, the most conservative pattern of ratings was obtained in the combination range $=0.80$ and midrange $=0.60$ [mean rating $\left(A_{1}\right)=25$, mean rating $\left(A_{2}\right)=42$ ]. Conversely, the pattern of ratings that was most favorable to Action $A_{1}$ was observed when range $=0.40$ and midrange $=0.30$ [mean rating $\left(A_{1}\right)=43$, mean rating $\left(A_{2}\right)=25$ ]. As the fine associated with $A_{3}$ increases $(y=3$ versus $y=2$ ) this action was rated as less attractive. All three factors were significant: range of probabilities $\left(F_{(6,446)}=8.19 ; P<0.05\right)$, Midrange of the probability intervals $\left(F_{(6,444)}=26.22 ; \quad P<0.05\right)$, and the two fines $\left(F_{(2,76)}=11.64 ; P<0.05\right)$. The only significant interaction we found was the 3 -way interaction between the range of the probabilities, their midrange, and the fines $\left(F_{(4,240)}=2.93 ; P<0.05\right)$. The interaction did not involve any cross-over and merely reflects slightly different rates of change in the various cases.

\subsubsection{Additional analysis of choices}

The MANOVA of normalized ratings reported in the previous section showed that the subjects in the Control group were most vagueness-averse, and that subjects in all groups were sensitive to the range and midrange of the probability intervals. This pattern was also reflected in the modal choices in the 18 cases; Action $A_{1}$ is the favorite in cases with low midranges and narrow ranges, and the safe action $\left(A_{2}\right)$ is favored in cases with high midranges and wide intervals. However, we did not find

\footnotetext{
7 The overall attractiveness of the various problems decreased monotonically as a function of the midrange of the probabilities - from a mean attractiveness of 11.6 for cases with the highest midrange of 0.60 , to a mean attractiveness of 13.5 for the cases with the lowest midrange of 0.30 . This difference is statistically significant $(F(3,223)=18.69 ; P<0.05)$

${ }^{8}$ The $F$ values reported in the text are those associated with Wilks' $\Lambda$.
} 
Table 1 Mean normalized ratings (and SDs) as a function of the decision aid, features of the probability interval and fines (Experiment 1)

\begin{tabular}{|c|c|c|c|}
\hline \multirow[t]{2}{*}{ Classification } & \multicolumn{3}{|l|}{ Action } \\
\hline & $A_{1}$ & $A_{2}$ & $A_{3}$ \\
\hline \multicolumn{4}{|l|}{ Decision aid } \\
\hline Control & 31 (12.6) & $\mathbf{3 5}$ (13.4) & $34(9.1)$ \\
\hline Display & 37 (13.0) & $31(9.9)$ & $32(8.9)$ \\
\hline Summary & 38 (10.6) & $29(11.1)$ & $34(7.7)$ \\
\hline \multicolumn{4}{|l|}{ Range } \\
\hline 40 & $41(10.5)$ & $26(9.1)$ & $33(8.5)$ \\
\hline 60 & 37 (11.6) & $29(10.2)$ & 34 (8.6) \\
\hline 80 & 39 (12.6) & 36 (12.6) & $34(8.7)$ \\
\hline 100 & $30(12.0)$ & 38 (12.9) & $33(8.7)$ \\
\hline \multicolumn{4}{|l|}{ Mid-range } \\
\hline 30 & $42(10.9)$ & $25(8.8)$ & 33 (8.9) \\
\hline 40 & 37 (11.5) & $30(10.2)$ & $34(8.3)$ \\
\hline 50 & 31 (11.7) & 35 (11.9) & $34(8.8)$ \\
\hline 60 & $35(10.9)$ & $42(13.0)$ & $34(8.7)$ \\
\hline \multicolumn{4}{|l|}{ Fines for $A_{3}$} \\
\hline$(2,5)$ & $35(12.1)$ & $31(12.1)$ & $35(8.6)$ \\
\hline$(3,4)$ & $36(12.9)$ & 32 (11.6) & $32(8.5)$ \\
\hline Overall & 35 & 31 & 33 \\
\hline
\end{tabular}

All numbers rounded to the nearest integer. Entries in each row add to 100. The highest rated action in each row in bold face; lowest ranked in italics

Parameters $e=\$ 10, f=\$ 4$

significant interactions between the decision aids and these features of the vague probabilities.

To address this issue that is relevant to $H 3$, we re-analyzed the data after combining the results of the two groups with decision aids (Summary and Display) and pooling actions $A_{1}$ and $A_{3}$. In this analysis we focus on the choices of the best action (rather than the ratings) to facilitate comparison across the various conditions. Figures 4 and 5 display the rate of choices of the safe action by DMs with, and without, access to decision aids, separately for each of the four distribution ranges (in Fig. 4), and for each of the four midranges (in Fig. 5). The figures highlight two patterns: (1) choice of the safe option increases monotonically as a function of the range and midrange of the vague interval and (2) this effect is reduced in the presence of decision aids. For each of these subsets we calculated the Odds Ratio (OR) of the 2 (Presence or absence of aid) $\times 2$ (action) table. All ORs are greater than 1 and 6 (out of 8 ) are significant $(P<0.05)$ indicating a higher tendency to select the safe action in the absence of decision aids. The overall OR (across all cases) is $1.64\left(\chi^{2}(1)=16.06, P<0.05\right)$. 


\section{Proportion of Choices of Safe Action With and Without}

Decision Aids as a Function of the Range of Probabilities

(Experiment 1)

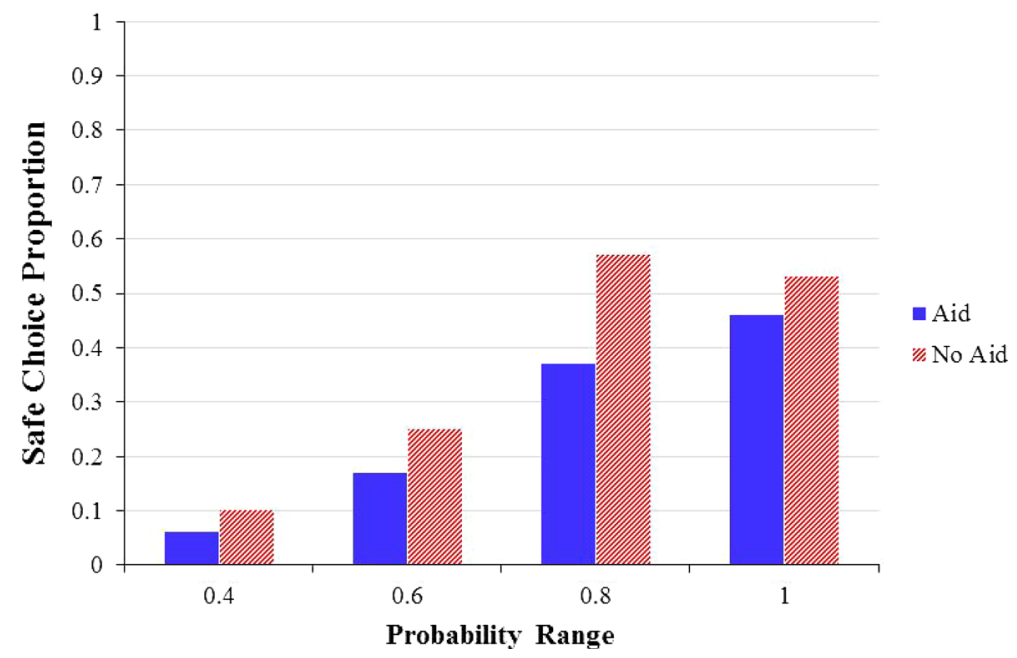

Fig. 4 Proportion of choices of the safe action as a function of the range of probabilities

\section{Proportion of Choices of Safe Action With and Without Decision Aids as a Function of the Mid-Range of the Probabilities (Experiment 1)}

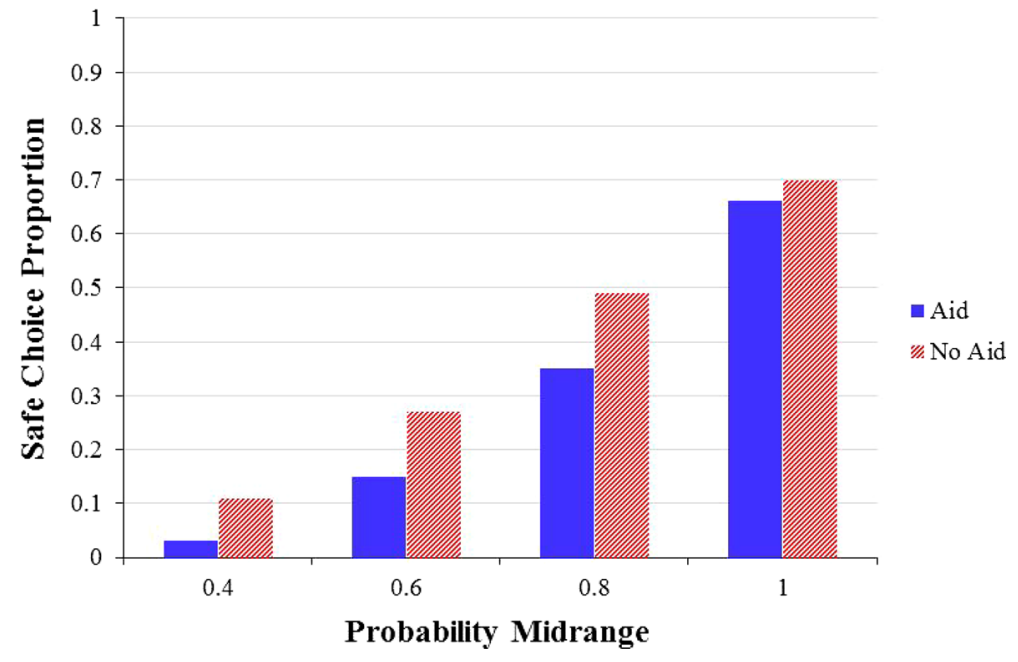

Fig. 5 Proportion of choices of the safe action as a function of the midrange of the probabilities

\subsubsection{Analysis of confidence ratings}

We submitted the DMs' subjective confidence ratings to a 4-way ANOVA with one between-subjects factor (the decision aid), and three within-subjects factors (range 
of the probability interval, midrange of the probability interval; the fines associated with action $A_{3}$ ). The mean confidence was 4.75 and there were significant differences between the four ranges of probabilities (ranging from 4.6 to 5.1) $\left(F_{(3,224)}=9.49 ; P<0.05\right)$, and the midranges of the four probability intervals (ranging from 4.7 to 5.1$)\left(F_{(3,223)}=13.84 ; P<0.05\right)$. The DMs' confidence increases as the range and midrange of the probability interval decrease, i.e., when the information is more precise and less aversive. However, there were no differences between the groups with the various decision aids.

\subsection{Summary and discussion}

The results support most of our hypotheses. Consistent with $H 1$, which was derived from the vagueness avoidance hypothesis, we found considerable support for the safe action-it was the highest rated action, and the modal choice, in the Control group as well as in many cases (e.g., highest levels of vagueness and highest midranges) across all groups. These include decision problems where the other actions offer superior outcomes. If, for example, one assumes a uniform distribution over the $\left[P_{\mathrm{L}}, P_{\mathrm{U}}\right]$ range in each urn (see Fox and Rottensterich 2003; Seale et al. 1995) $A_{2}$ has the highest expected value in only one case (range $=0.8$, midrange $=0.6, y=\$ 3$ ), yet it was the modal choice for 7 of the 18 urns!

Confirming our prediction (and the vagueness avoidance hypothesis) the tendency to choose the safe action increased monotonically in the range of the probability interval and its midrange (replicating Kramer and Budescu 2005). When DMs moved away from the safe action, the beneficiary was, typically, action, $A_{1}$. The attractiveness of, and the tendency to choose, this action increased monotonically as we reduced the range and the midrange of the probability interval. The support for the intermediate action, $A_{3}$, was relatively stable across all conditions.

The tendency to choose the "riskier" actions, $A_{1}$ and $A_{3}$, was considerably higher when the DM had access to either decision aid, confirming $H 2$, but we did not detect differences between the two aids. $H 4$ predicted that the DM's confidence ratings would, essentially, mimic their attitude to vagueness. This prediction was supported only partially: Confidence was, indeed, higher as the range and midrange of the probability interval was reduced (those cases that caused people to choose the riskier action). Contrary to our expectations, confidence was not affected by the presence of decision aids although, interestingly, in the Control group confidence was highest when choosing the safe action, $A_{2}$, whereas in the presence of the decision aids the confidence peaked when the action, $A_{1}$, was chosen.

A somewhat surprising result was the low rate of information purchase. Subjects used this option in a reasonable fashion (i.e., more often in the absence of decision aids, and for high levels of vagueness). This observation invalidates the possibility that they did not understand this option, or the procedure. In retrospect, it appears that this result is due to the overpricing of the information.

One intriguing result of the first study is that the differences between the two decision aids (predicted by H3) were not fully confirmed - the results of the DMs who were exposed to Summary and Display were highly similar in most respects. The next two studies investigate further the two aids. 


\section{Experiment 2: subjects prefer the Display over the Summary aid}

We compared the subjects' performance with two distinct aids that differ along several dimensions: They use different formats (numerical in Summary versus graphical in Display), vary with respect to the nature of the user interface with the aid and the ability to Control it, and one (Summary) offers a clear prescription for action, while the other (Display) does not. Thus, one cannot attribute differences between the two conditions to a single attribute that can be easily controlled or manipulated. The two aids were selected to be best representations of diametrically opposed approaches to imprecision—one seeks to minimize and (almost suppress) it and the other highlights its effects. Interestingly, the first experiment revealed little difference in subjects' choices using the Summary and Display aids. This second experiment goes beyond the subjects' choices and aims to determine whether DMs prefer using one of the two aids.

To this end we first exposed the DMs to both decision aids and subsequently offered them an opportunity to choose one of them for additional problems. Recall that the expected value calculator (Summary) rank orders the actions contingent on the hypothetical probability distributions over the $\left[P_{\mathrm{L}}, P_{\mathrm{U}}\right]$ range, specified by the DM. The major advantage of the Summary aid is that it offers an unambiguous prescription for choice. Its major disadvantage is that it asks DMs to specify probability distribution(s) over $\left[P_{\mathrm{L}}, P_{\mathrm{U}}\right]$ when there exists little information and little reason to depart from an ignorance prior. The Display aid highlights the imprecision. The major advantages of this aid are that (a) it does not require additional input from the DM and (b) it illuminates the ranking of the actions over the full range of imprecise probability $\left[P_{\mathrm{L}}, P_{\mathrm{U}}\right]$. In terms of Sanfey and Hastie's (1998) analysis it can be argued that Display is more similar to (and compatible) with the nature of the data. Its major disadvantage is that it does not offer an unambiguous prescription for choice. We predict that the salience of the imprecision and the ease of use will dominate, and the subjects will reveal a general preference for Display over Summary.

\subsection{Method}

\subsubsection{Participants}

Thirty-two students at the University of Illinois at Urbana-Champaign (7 men, 25 women) were recruited using the same procedure as Experiment 1.

\subsubsection{Design}

The dependent variables of interest were choice of decision aid and consistency in repeated decisions. Subjects were randomly assigned to one of four groups defined by crossing the order of presentation of the aids and the sets of urns. After subjects were exposed to both decision aids, they were allowed to choose which aid to use for each of the final nine trials. Each subject made decisions regarding 15 total urns. 
In all cases we fixed the endowment parameter, $e$, at $\$ 20$. We manipulated the parameters for actions $A_{2}$ and $A_{3}$.

To summarize, we ran four groups defined by counterbalancing (a) the first two urn sets and (b) the decision aids. Each subject judged 15 urns (with 6 urns repeated).

\subsubsection{Procedure}

The procedure was similar to Experiment 1 in most respects. There were 15 opaque urns that were divided into three different sets. The first two sets consisted of three urns each. They were matched in terms of the probability intervals and the $(\$ y+\$ z)$ values. The third set consisted of nine urns (duplicates of the original six and three new ones). Each participant was exposed to all 15 urns but in different orders and with different aids. The parameters of the urns used are listed in Table A2 in the Appendix. Each subject used one of the decision aids (Display or Summary) for the first set and the other decision aid (Summary or Display, respectively) for the second set. The final set of nine urns offered the subject the option of choosing one of the decisions aids on each trial. Within each set the order of presentation of the urns was randomized.

Participants were informed that one of the 15 urns would be selected to determine their final payoff and advised to make decisions for each urn as if they were going to play the gamble for cash. DMs were informed about the contents of the urn $\left(P_{\mathrm{L}}\right.$ and $\left.P_{\mathrm{U}}\right)$, and the monetary parameters $(e, f, y$, and $z)$ associated with the various actions $\left(A_{1}, A_{2}\right.$, and $\left.A_{3}\right)$ for each urn (the option to purchase information was excluded in this study). Then, they turned to the computer and keyed in the parameters to use the decision aid depending on the condition in which they were. After consulting their aids the participants rated the attractiveness of each action on a 7-point scale, selected one of the actions, and rated their confidence in the choice (using a 7-point scale).

After all decisions were made, one of the urns from the third group was selected at random and played depending on the action the subject picked for that urn. Finally, subjects were paid and dismissed. The experiment lasted about one hour and subjects earned between $\$ 8$ and $\$ 20$. The mean payoff was $\$ 13.80$ $($ median $=\$ 13, \mathrm{SD}=\$ 4.18)$.

\subsection{Results}

\subsubsection{Preferences for decision aids}

We focus on the last group of urns where the subjects could choose an aid on nine separate occasions. The overwhelming majority of these choices $(75 \%)$ favored the Display aid. This preference for the Display was observed for a majority of individual judges: 23 of them (72\%) select the Display at least 8 times (out of 9), and only 5 people $(16 \%$ ) have a $4 / 5$ or $5 / 4$ split indicating relative indifference between the two aids. 


\subsubsection{Consistency of repeated decisions}

Recall that six decisions were repeated (once with a prescribed aid and once with the aid chosen by the DM). Table 2 cross-classifies the repeated choices for these 6 urns across all 32 subjects. The overall level of agreement between the two replications (i.e., the entries along the diagonal) is $48 \%$, and the corresponding Cohen's $\kappa$ is 0.18 . This level may appear low but it is consistent with other studies (e.g., Loomes et al. 2010; Por and Budescu 2013). The level of agreement was, practically, identical in the 98 cases where subjects used the same aid twice and in the 94 cases where they switched on the second presentation. Not surprisingly, the highest level of consistency of choice was found (in the small number of cases) when DMs used the Summary twice, because this aid offers an unequivocal prescription for action (which was almost always followed).

\subsection{Summary and discussion}

The two decision aids differ along several dimensions such as their format and the nature of the user interface with the aid. The results indicate a strong preference for the Display aid, as predicted. This preference suggests that the simplicity of the aid, its completeness, and the fact that it does not call for any input from the DM were sufficient to overcome what can be seen as its major drawback, namely the lack of an explicit and unambiguous recommendation for action. This suggests that, contrary to the "common wisdom," DMs do not necessarily prefer precision (see also Du et al. 2011 for similar results in a financial context).

Given that we used a subset of the same problems twice we were in a position to test the consistency of the choices (and ratings) across replications. Overall, subjects achieved a respectable, but not exceptional, level of consistency that was equal for those who used the same aid twice and for those who switched highlighting, again, the similarity between the two aids demonstrated in the previous experiment.

\section{Experiment 3: the nature of the decision aid influences the ranking of actions}

We hypothesized (H3) that exposure to the Summary aid would induce preferences for the actions with higher expected values, assuming a non-informative prior distribution over the probability interval $\left[P_{\mathrm{L}}, P_{\mathrm{U}}\right]$, while exposure to the Display aid

Table 2 Cross-classification of choices in the repeated urns (Experiment 2)

\begin{tabular}{lllll}
\hline Original choice & \multicolumn{2}{l}{ Second (optional aid) choice } & Total \\
\cline { 2 - 4 } & $A_{1}$ & $A_{2}$ & $A_{3}$ & \\
\hline$A_{1}$ & 11 & 18 & 22 & $51(26 \%)$ \\
$A_{2}$ & 9 & 54 & 19 & $82(43 \%)$ \\
$A_{3}$ & 7 & 26 & 26 & $59(31 \%)$ \\
Total & $27(14 \%)$ & $98(51 \%)$ & $67(35 \%)$ & 192 \\
\hline
\end{tabular}


will cause DMs to favor the actions that are best over the largest segment of the relevant probability range, but the first experiment did not support this hypothesis. This study is an additional effort to test this prediction with a different set of problems designed to highlight the differences between the two. Decision parameters were chosen so that different actions would be the best when using the expected value and the interval of dominance criteria. We expect that DMs would choose in line with the approach to vagueness emphasized by the two aids: DMs using the Summary (which calculates EVs) would prefer the action with the best expected value, while DMs with access to the Display (which shows the relations between the various actions across all probabilities) would favor the action that is superior to the others in a majority of cases (i.e., for most values of $P$ in the relevant range). More specifically, we expect that among those DMs using the Display action $A_{1}\left(A_{3}\right)$ would be rated highest (lowest), with $A_{2}$ in between. Conversely, for the DMs using the Summary, the prediction is that action $A_{3}\left(A_{1}\right)$ would be rated highest (lowest), with $A_{2}$ in between.

\subsection{Method}

\subsubsection{Participants}

Thirty-nine students at the University of Illinois at Urbana-Champaign (16 men, 23 women) were recruited using the same procedure as in the other studies.

\subsubsection{Design}

Participants were randomly assigned to one of three experimental conditions (Display, Summary, and Control). For all 12 urns we fixed the endowment parameter, $e$, at $\$ 20$. The voluntary fine for the safe action took two values $(f=\$ 8$ or $\$ 10)$. We also manipulated the parameters of action $A_{3}$. We used several pairs of values for the cost of insurance $(y)$, and the additional loss $(z)$, and three different levels of vagueness. The parameters for all 12 cases are displayed in the Appendix. In all 12 cases examined, $\operatorname{EV}\left(A_{3}\right)>\operatorname{EV}\left(A_{2}\right)>\operatorname{EV}\left(A_{1}\right)$, but $\operatorname{Top}\left(A_{3}\right)<\operatorname{Top}\left(A_{2}\right)$ $<\operatorname{Top}\left(A_{1}\right)$, where $\operatorname{Top}\left(A_{i}\right)$ is the percent of the range for which $A_{i}$ has the highest EV.

To summarize, the experiment consisted of three groups of participants defined by the different decision aids. Each subject judged 12 urns defined by (a) three levels of vagueness (b) two levels of safe fines, f, and (c) 12 different pairs of $y$ and $z$.

\subsubsection{Procedure}

Subjects were run individually. Upon arrival to the laboratory they were randomly assigned to one of the experimental conditions, were handed the relevant instructions, signed the informed consent form, and proceeded to make 12 independent decisions regarding urns filled with colored balls.

The procedure and decision tasks were identical in most respects to Experiment 1. For each of the 12 urns the subjects keyed in the parameters of the decision task 
$\left(P_{\mathrm{L}}, P_{\mathrm{U}}, e, f, y, z\right)$, consulted their aids, and then rated the attractiveness of each action on a 7-point scale, selected one of the actions, and rated their confidence using a 7-point scale.

The earnings were determined using one urn chosen (at random) at the conclusion of the study. The participants' recorded choice determined the appropriate random draws from the urn. The experiment lasted about one hour. Subjects earned between $\$ 8$ and $\$ 20$. The mean payoff was $\$ 12.50$ (median $=\$ 10$, $\mathrm{SD}=\$ 4.4)$.

\subsection{Results}

Some of the urns used in Experiment 2 are identical to some of the urns used in the present study (see Table A2 in the Appendix). To increase the power of our analysis we used these data as well. ${ }^{9}$ More specifically, we complemented the results of the 39 subjects in Study 3, with all the choices made by the 32 subjects in Study 2 for the same urns, in the first stage (i.e., in the first 3 trials of the study before the subjects were exposed to other decision aids).

\subsubsection{Analysis of ratings in the presence of decision aids}

The safe action, $A_{2}$, was rated the highest at the group level, and is also the modal individual pattern (around $40 \%$ of DMs), confirming, again the vagueness avoidance hypothesis. We predicted that action $A_{1}\left(A_{3}\right)$ will be rated higher (lower), by those using the Display (Summary) aid and, conversely, we expected those using Summary (Display) to rate $A_{3}\left(A_{1}\right)$ higher (lower). Table 3 shows how often $A_{1}$ was rated higher (lower) than $A_{3}$ in the presence of the two decision aids (cases with tied rankings are excluded). There are more cases where $A_{3}$ is rated higher than $A_{1}$, overall (71\%) and in each condition. However, the balance of cases rating $A_{1}$ higher or lower than $A_{3}$ is in line with our prediction: In the Display condition $A_{1}$ is rated as more attractive in $60 / 162=0.37$ instances, whereas in Summary the rate is $37 / 170=0.22$. The difference is statistically significant $\left(\chi_{(1)}^{2}=9.36, P<0.05\right)$.

Finally, we calculated the difference between the mean (normalized) ratings of $A_{1}$ and $A_{3}$ for the various decision problems and regressed this difference (a proxy for the strength of preference for the risky action) on the various features of the decision problems and a binary variable indicating which decision aid (Summary or Display) was used. This binary variable was the single best predictor of the difference $\left(r=0.41 ; t_{(24)}=2.41, P<0.05\right)$. Overall, the regression achieved a good fit $\left(R_{(\mathrm{adj})}^{2}=0.45 ; F_{(5,20)}=5.02, P<0.05\right)$. Dominance analysis (Azen and Budescu 2003) shows that the nature of the decision aid accounts for $31 \%$ of this fit, making it the second most important predictor (it is surpassed only by $\$ y$, the cost of insurance associated with $A_{3}$, that accounts for $32 \%$ of the variance).

\footnotetext{
9 The two studies were ran only a few weeks apart in the same lab, by the same research assistants, and the subjects were recruited from the same population using identical procedures.
} 
Table 3 Proportion of cases with different patterns of ratings of $A_{1}$ and $A_{3}$ as a function of the decision aid (Experiments 2 and 3)

\begin{tabular}{llll}
\hline & Display & Summary & Total \\
\hline Rating $\left(A_{1}\right)>$ Rating $\left(A_{3}\right)$ & 60 & 37 & $97(29 \%)$ \\
Rating $\left(A_{1}\right)<$ Rating $\left(A_{3}\right)$ & 102 & 133 & $235(71 \%)$ \\
Total & $162(49 \%)$ & $170(51 \%)$ & 332 \\
\hline
\end{tabular}

Indeterminate (tied) cases excluded

\subsection{Summary and discussion}

We predicted a systematic relationship between the nature of the decision aid and the DM's choices: We expected exposure to the Summary aid to induce preferences for the actions with higher expected values, while exposure to the Display aid to cause DMs to favor the actions that dominated the others for a largest portion of the relevant probability range. To test this prediction we focused on a subset of cases in which DMs using the Display aid were expected to rate $A_{1}$ highest, and $A_{3}$ lowest with $A_{2}$ in between, while those using the Summary were expected to endorse the opposite rating. In most cases the DMs favored the safe $\left(A_{2}\right)$ action, confirming the vagueness avoidance hypothesis, but not supporting the strong version of our prediction regarding the two decision aids. Secondary analyses focusing on the DMs' ratings of actions $A_{1}$ and $A_{3}$ confirmed our hypothesis: Subjects who had access to different decision aids rated the various actions differently. These subtle differences are consistent with the predictions of $H 3$.

\section{Identifying decision rules for decisions with vague probabilities}

In this section we tackle what may be the most interesting problem from a behavioral decision theoretical point of view, namely identifying what type of models best describe the DM's decisions. Since there is no normative model for the problem of decision with imprecise probabilities (e.g., Etner et al. 2012; Manski 2010; Troffaes 2007), we compared a variety of models proposed for decisions under ignorance (probabilities unknown) and for decisions under risk (probabilities known or estimable) (e.g., French 1986; Luce and Raiffa 1954). The question of the appropriate decision model in the context of climate change was discussed recently by Kunreuther et al. 2013). The questions we address are (1) which class of models fits best decisions in this gray area, and (2) how is the fit of the various classes of models associated with the availability of decision aids. Our hypothesis (H5) is that we will find groups of DMs that use, primarily, models of decisions under ignorance that ignore probabilities and others that will act in line with a Subjective Expected Value (SEV) model with probabilities inferred from the decision.

To keep the comparison fair and relatively simple we avoided any models that require estimation of individual parameters (e.g., Hurwicz's optimism-pessimism criterion). Table 4 lists the nine models we compared. They fit into four 
Table 4 List of decision models being compared and their key characteristics

\begin{tabular}{llll}
\hline Model & Model & Key index & Choice criterion \\
\hline 1 & Maxi-min & Lowest outcome for each action & Maximal \\
2 & Maxi-max & Highest outcome for each action & Maximal \\
3 & Mini-max regret & Highest regret for each action & Minimal \\
4 & Mini-min regret & Lowest regret for each action & Minimal \\
5 & $P^{(\mathrm{U})}=P_{\mathrm{U}} /\left(1+P_{\mathrm{U}}-P_{\mathrm{L}}\right)$ & Proportional to the upper bound & Maximize EV \\
6 & $P^{(\mathrm{L})}=P_{\mathrm{L}} /\left(1+\mathrm{P}_{\mathrm{L}}-\mathrm{P}_{\mathrm{U}}\right)$ & Proportion to the lower bound & Maximize EV \\
7 & $P^{(\mathrm{M})}=\left(P_{\mathrm{U}}+P_{\mathrm{L}}\right) / 2$ & Mid-range & Maximize EV \\
8 & Laplace & Expected value for each action & Highest EV \\
9 & Starr & Expected value for each action & Highest EV for most \\
& & & probabilities \\
\hline
\end{tabular}

qualitatively distinct classes: The first class involves maximization of the minimal outcome (maxi-min) and of the maximal outcome (maxi-max) without any considerations of probabilities. The second class takes a similar approach, but in the regret metric-minimization ${ }^{10}$ of the maximal regret (mini-max regret) and of the minimal regret (mini-min regret), again without any concern for probabilities. These two classes represent the standard approaches to decisions under ignorance. The third class involves three versions of a SEV model that vary only in terms of the (estimate of the) probability they use. They involve probabilities that are proportional to (a) the lower bound of the range (essentially, disregarding the balls of unknown color, as if they do not matter), (b) the upper bound of the range (counting the balls of unknown color twice, as if they provide simultaneous support to both colors), and (c) the mid-range (assuming a uniform prior over the range). The last class consists of two methods of finding the action with the highest expected value across the entire range of probabilities. The first (Laplace) assumes a uniform prior over the range and computes the posterior expected value, and the second (Starr) considers an action to be chosen if it has the highest expectation for the largest fraction of the range of imprecision.

We analyzed 2,346 choices involving 11 distinct probability ranges across all three experiments. ${ }^{11}$ Appendix Table A3 shows the decisions being analyzed. We compared the subjects' choices with the predictions of the nine models and evaluated performance by two measures: $P_{\mathrm{c}}$, the proportion of correct predictions, and PRE, the Proportional Reduction of Error, which is defined as PRE $=\left[P_{\mathrm{c}}-\right.$ $E\left(P_{\mathrm{c}} \mid\right.$ Chance $\left.)\right] /\left[1-E\left(P_{\mathrm{c}} \mid\right.\right.$ Chance $\left.)\right]$. PRE adjusts the observed $P_{\mathrm{c}}$ for the rate of correct predictions expected by chance alone. In most cases $E\left(P_{\mathrm{c}} \mid\right.$ Chance $)=1 / 3$ (since there are three possible actions), but in some instances two actions lead to identical outcomes, and we set $E\left(P_{\mathrm{c}} \mid\right.$ Chance $)=1 / 2$.

Two models dominate the others (see Table 5) - the highly conservative, maximin model that ignores the probabilities altogether and focuses on the worst-case

\footnotetext{
${ }^{10}$ Recall that regrets are, by definition, non-positive and the DMs seek to minimize them.

${ }^{11}$ We only excluded some of the decisions form Experiment 1 made by subjects who purchased informations.
} 
Table 5 Fit measures of the best two models (individually and jointly) (Experiments 1, 2 and 3)

\begin{tabular}{lllll}
\hline Proportion of correct predictions (and PRE) & & \\
\hline Model & \multicolumn{4}{l}{ Group (decision aid) } \\
\cline { 2 - 5 } & Control & Display & Summary & Total \\
\hline Maxi-Min & $51(26)$ & $49(23)$ & $46(19)$ & $48(22)$ \\
Probability $=P^{(\mathrm{L})}$ & $44(17)$ & $51(27)$ & $53(29)$ & $50(25)$ \\
Maxi-Min OR probability $=P^{(\mathrm{L})}$ & $66(49)$ & $64(46)$ & $64(46)$ & $64(46)$ \\
\hline
\end{tabular}

scenario, and a version of SEV that estimates the probability of the target event using the lower bound of the range, $P_{\mathrm{L}}$. This version of SEV ignores the balls of unknown colors and is consistent with the notion that the observed balls are a random sample of all the balls, so one can use them to estimate the total (unknown) proportion of red balls in the urn.

Both models fit the choices equally well at the aggregate level: each predicts correctly about half of the choices and jointly they predict about two-thirds of them. The results hold also at the individual level: $42 \%$ of the subjects are best fit by the maxi-min model and $29 \%$ are best fit by the SEV with $P_{\mathrm{L}}$, and the average PRE is the same (0.37) in both subgroups.

The most interesting observation is that the fit of the models is related to the decision aids used: the conservative maxi-min predicts better than SEV in the Control group, but the opposite pattern emerges in the presence of either of the decision aids (Display and Summary) that highlight the probabilities and cause people to pay more attention to them. In the same spirit, the fit of some of the other models (not presented in Table 5) is also sensitive to this manipulation. For example, the Laplace model fits considerably better in the presence of either decision aid than in the Control group.

Figure 6 presents the performance of the two best models as a function of the vagueness (the range of probability interval) of the decision problem. The left panel is based on all the data and the right one is based on the Control group alone. The figure illustrates clearly that whereas the prediction validity of the maxi-min model is relatively constant across all cases, the fit of the SEV model is inversely related to the level of vagueness. In other words, as the level of inherent vagueness in the decision problem increases, the less likely are DMs to approach the problem as one of decisions under risk (see also Hey et al. 2010, for a similar pattern).

\section{Summary and general discussion}

\subsection{Summary of the results}

We reported results from three experiments designed to study how DMs evaluate and choose among actions involving imprecise probabilities. If one knew the actual probability, $P$, of the undesirable event (two red balls) the actions would be 
All

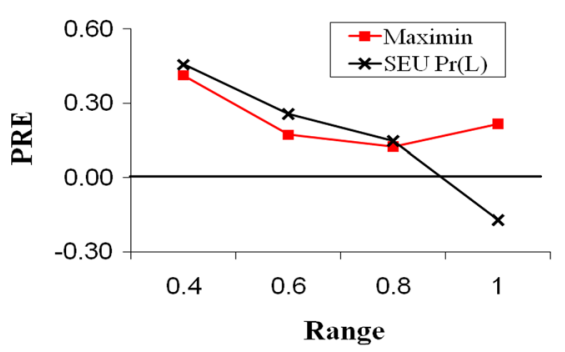

Control

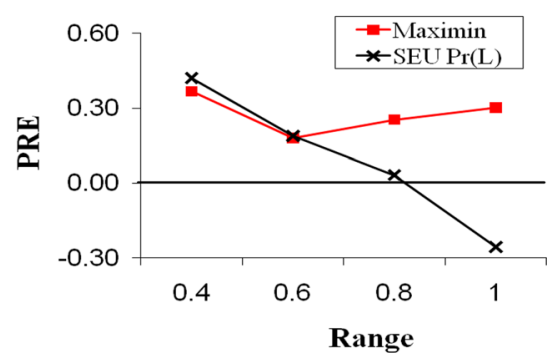

Fig. 6 PRE of the best two models as a function of the level of imprecision

described as "risky", "safe", and "reduced risk". In this case, however, all decisions were made in the presence of irreducible vague probabilities which were presented in various ways. The probabilities were characterized by specifying their (sharp) lower and upper bounds. Our design was as "hands on" as possible, as the subjects literally counted and filled the urns with balls of different colors to assure that they understood the level of imprecision. The probability ranges and the various payoffs were selected such that each of the three actions was superior to the other two for some subset of the relevant range so the DMs had to invoke and apply rules that trade off the specific outcomes and the vague probabilities. The second goal of the work was to test the effects of various presentation modes (decision aids) on the DMs' responses.

There is a voluminous theoretical and empirical literature on the effects of vagueness (imprecision) on decisions, but the design of our study is quite unique. The vast majority of the existing literature that was inspired by Ellsberg's (1961) paper has followed his lead by focusing on the direct effect of different sources of uncertainty (Abdellaoui et al. 2011). Researchers have studied how DMs choose between options or actions that are identical in all respects but involve different source functions (i.e., one precise and one imprecise). In a sharp departure from this tradition, we consider the case of single source function which is, invariably, imprecise. We study how people evaluate, and choose among, several actions that are available to them in the presence of (identical) irreducible vague probabilities. There are cases where one needs to choose between various sources (e.g., choosing between two advisors who vary in their precision: adviser A forecasts that the probability of the market gaining $X \%$ in the next 6 months is 0.05 , while Advisor B estimates this probability to be between 0.02 and 0.09 ), but our setup captures the much more common problem of having to choose one action in an environment characterized by irreducible imprecision.

More specifically, our thinking was guided by the climate change dilemma: Given incomplete and imperfect information available, the estimates of the probability of the effects of climate change are often imprecise. In this context would DMs choose to do nothing $\left(A_{1}\right)$, reduce emissions drastically at a high cost $\left(A_{2}\right)$, or take intermediate (less effective but also less costly) actions? Another implication of the climate change motivation is that we focused on the domain of 
losses. We realize that there are locations with local circumstances where some may benefit from the effects of climate change (e.g., it may be easier and cheaper to mine for, and transport, minerals in Siberia in warmer temperatures), but at the global level, the consequences of climate change are undesirable and are typically encoded and treated as losses. There is some experimental evidence that loss aversion may affect attitudes to, and reaction to, vagueness (Trautmann et al. 2011), but this observation does not affect our key findings which, as mentioned earlier, apply to fixed levels of vagueness and are all in the domain of losses. Thus, all the comparisons between the groups are valid. We do caution, however, against generalizing these findings to the domain of gains without additional empirical studies.

The results confirmed most of our predictions: (a) consistent with the vagueness avoidance hypothesis, the DMs displayed systematic preferences_-as measured by both the normalized ratings and the choices-for the safe action. In some cases this choice came at a clear premium. The preference for the safe action increased as a function of the level of vagueness and the probability of the undesirable outcomes. (b) Decisions were affected by access to decision aids. The aids significantly increased the DMs' preference for the riskier actions. Although the choices made in the presence of the two decision aids were similar in many respects, we found in Experiment 3 evidence for a subtle interaction between the nature of the decision aid used and the DMs' decisions. More specifically, exposure to the expected value calculator in the Summary aid induced higher preferences for the actions with higher expected values, and the Display aid caused DMs to favor more actions that were superior for the largest portion of the relevant range of the probabilities, confirming that the salience of the entire range of probabilities affected the way the problem was processed (Fiedler and Unkelbach 2011; Kleinmuntz and Schkade 1993; Sanfey and Hastie 1998). This can also be associated, at least in part, with the level of compatibility between the decision aids and the decision (e.g., the EV calculator inducing the choice of the action with the highest EV).

In the second experiment the DMs were exposed to both aids and were allowed to choose the one they preferred. A clear majority expressed a preference for the Display aid which shows the expected values of all actions for all probabilities and explicitly identifies the regions where each of the actions is superior. This preference is quite striking since, unlike the Summary, the Display does not provide a direct guide to action and does not resolve the vagueness (if anything, it highlights it). As such, it flies in the face of the common wisdom, based in part on Ellsberg's paradox, that DMs always prefer precise information. The recent paper by Du et al. (2011) showed a similar pattern in the DMs' preferences for vague forecasts over more precise ones when evaluating financial forecasts. Du et al. (2011) found that the vague forecasts were perceived as more informative, reliable, and credible. More importantly, they showed that DMs have clear expectations about the level of precisions that make sense in various domains (e.g., high precision in medicine and lower in financial and political forecasts) and they seem to favor forecasts whose precision is congruent with their expectations.

We analyzed almost 2,500 choices made in the three experiments to evaluate whether decisions under vagueness-so prevalent in real life (e.g., medical, 
environmental, political, and insurance decisions) - are best modeled by rules developed for decisions under ignorance or those for decisions under risk. We found that DMs are as puzzled by these choices as decision theorists and do not favor one class of models consistently. We found support for the conservative maxi-min criterion that ignores probabilities but, interestingly, a version of SEV with probabilities inferred from the partial information available (the visible balls) also performed well, especially for lower levels of vagueness, suggesting that these cases are perceived as closer to classical decisions under risk. The fact that the best fitting models for the DMs' choices also varied as a function of the decision aids lends further support to our claim about the DMs' sensitivity to the presentation format.

\subsection{Some implications of the results}

One major motivation of this work is to contribute to ongoing discussions about how to best characterize and present scientific and economic information for policy makers addressing climate change and other policy areas where probabilistic information is irreducibly imprecise. We conclude with some insights that our work might offer towards these questions.

We found decision aids made a significant difference in subjects' choices. Without such aids, subjects were far more likely to choose the safe action, but with them subjects exhibited greater willingness to trade-off imprecision and risks. Subjects displayed a stronger inclination towards actions that accept increased risks in return for potentially larger gains. This effect varied across the levels of vagueness suggesting that the more vague the underlying information, the greater the impact well-crafted, formal decision aids may have on the willingness of policy makers to take a more nuanced view of the best balance among the risks they face.

Our two aids represent different views about the appropriate presentation of information about irreducible uncertainty to decision-makers. The Summary aid focused on comparing expected values of alternative decisions contingent on bestestimates of the imprecise probability distributions while the Display aid compares the performance of options over the entire range of plausible probabilities. Exposure to the graphical displays caused more subjects to favor the action that was perceived as superior for larger portions of the probability range, compared with subjects who had access to an expected value calculator. This supports the thesis of Kleinmuntz and Schkade (1993) that various presentation modes can trigger different decision processes, and Sanfey and Hastie's (1998) observation that such triggering occurs though highlighting, or increasing the salience of, various aspects of the information. These two results - that alternative aids highlight alternative attributes of a decision and that aids which summarize the entire range of plausible probabilities can affect (albeit slightly in our experiments) the decision rules usedillustrate how the design of decision aids may affect the risks on which users focus and the level of risk they accept. When presenting forecasts to decision makers, it is important to convey the level of (im)precision that warranted by the evidence used to generate those forecasts (e.g., Budescu and Wallsten 1987). Analogously, our results suggest that future research might usefully examine proper design principles 
for decision aids in situations with significant imprecision and potentially different views on the most appropriate ways to balance among various risks.

When offered a choice between the two displays, most participants opted for the visual display that presents the performance of the various actions for all probabilities. Although the precise source of the preference is not fully explained, this pattern supports Sanfey and Hastie's (1998) claims that the key factors are the salience/prominence of the information and the similarity/compatibility between the nature of the data and the displays. Our findings also support Du et al. (2011) emphasis on the congruence between the underlying nature and sources of uncertainty and its communication, and are also consistent with the effort-accuracy tradeoff underlying the "adaptive DM" view (e.g., Payne et al. 1993). The Display involves minimal input and effort on the part of the DM and, because of its completeness, it also conveys the impression of allowing one to achieve maximal accuracy. On the other hand, the Summary calls for active involvement (and a higher effort) on the part of the DM, in the form of specification of the basic distribution(s) to be considered. And, unless one is highly confident that he/she has specified the right distribution(s), there will always be lingering doubts about the accuracy of the prescriptions. In terms of the recent analysis of Abdellaoui et al. (2011) one could also speculate that various presentation modes encourage people to analyze the problem differently in terms of the sources of uncertainty.

Our results also have implications for ongoing debates on the appropriate decision criteria to use in climate policy analyses. While most such analyses employ an expected utility or cost effectiveness criterion [America's Climate Choices (Liverman and Raven 2010)], the debate regarding the most appropriate criteria for comparing alternative policies is not settled in the literature (Hof et al. 2010; Kunreuther et al. 2013). Some studies have investigated the benefits of the minimax regret decision criteria for abatement decisions because it only requires estimates of worst case outcomes (Froyn 2005; Hof et al. 2010; Loulou and Kanudia 1999; Van den Bergh, 2004). Other studies examine various robustness criteria (Dessai and Hulme 2007; Wilby and Dessai 2010; Brown 2011; Reeder and Ranger 2011; Hall et al. 2012), some of which balance between mini-max regret and expected utility (Lempert and Collins 2007). Many of these studies aim to supply trade-off curves and other information that may aid in decision making, rather than suggest specific choices that should be made.

The choice of decision criteria in climate policy analysis depends on many factors, but it is nonetheless useful to understand the types of criteria decision makers might actually use when faced with various types of uncertainty. While they lack much of the specific context of actual policy decisions, our studies can provide insight into such questions because their decision problems do mimic some of the same tradeoffs faced by policy makers employing economic cost-benefit analysis to make decisions about carbon abatement (Lange and Treich 2008). Our results suggest that it is the most conservative maxi-min, rather than the mini-max regret criterion, that accounts for individual choice when faced with deep uncertainties and a similar set of actions in a context free setting. More broadly, our results support the value of non-standard decision criteria (i.e., not SEU) in the modeling of economic policy under deep uncertainty. 
These laboratory experiments also provide a useful complement to field evaluations examining how alternative decision aids affect policy-makers faced with deeply uncertain conditions. For example, these experiments helped inform the design and interpretation of evaluations used in a series of workshops held with managers, local elected officials, and constituents of Southern California's Inland Empire Utilities Agency, a water wholesaler serving about one million residents. The workshops presented analyses examining whether and how potential impacts from climate change should cause the agency to modify their then current longrange water management plans (Groves et al. 2007). Uncertain forecasts from climate, water management, and economic models were summarized in alternative decision aids - one set analogous to the Display aid, and another set analogous to the Summary aid-and survey instruments used to determine the extent to which the alternative aids affected the participants' judgments and preferences among alternative policy choices, and the participants' preferences among the aids. These workshops are not directly comparable with the experiments, for reasons including small sample sizes and the political necessity for the hosting agency to invite all participants to view all the information from all the available decision aids. Nonetheless, the workshop findings proved roughly consistent with the experiments. Workshop participants reported that the aids analogous to the Display contained more valuable information for planning purposes, but seemed more difficult to understand and explain to others than the aids analogous to the Summary. ${ }^{12}$

These workshops and experiments only begin to inform any judgments about the extent to which one presentation mode is superior to the other in any particular decision context. But the results do provide important guidance for future research about effective presentation of deeply uncertain information to decision makers. For instance, our study suggests it may be useful to consider decision aids that combine the desirable features of the two formats compared here. More extensive sensitivity analysis might improve confidence in the Summary aid, allowing DMs to test the invariance of the recommendations across a range of distributions. An optional Summary calculator might accompany the Display aid, the former using the DM's, or outside experts', specifications of probability distributions over the $\left[P_{\mathrm{L}}, P_{\mathrm{U}}\right]$ range. More broadly, future studies should examine how alternative decision aids affect choices embedded in content specific contexts, as opposed to abstract contexts. Nonetheless, this study does suggest that unavoidable and irreducible imprecision may enhance the relevance and importance of effective decision aids and poses unique challenges that should be considered in their design.

Acknowledgments This work was supported by the National Science Foundation under Grants SES 0345925 and SES 1049208. The opinions, findings and conclusions or recommendations expressed in this paper are those of the authors and do not necessarily reflect the views of the funding agency. We thank three anonymous reviewers for their useful and constructive comments. We are grateful to Alina Kobzarev and Ronnie Berg for assistance with data collection and to James Englert for programming the experiments.

\footnotetext{
12 IEUA did subsequently modify their water management plans in the face of deeply uncertain climate forecasts, attributing their actions in part to information presented in the workshops (Atwater 2008).
} 


\section{References}

Abdellaoui M, Baillon A, Placido L, Wakker PP (2011) The rich domain of uncertainty: source functions and their experimental implementation. Am Econ Rev 101:695-723

Arrhenius S (1896) Über den Einfluss des atmosphärischen Kohlensäuregehaltes auf die Temperatur der Erdoberfläche, Bihang Till K. Svenska Vet.-Akad. Handlingar 1:1-102

Arunachalam V, Pei B, Steinbart P (2002) Impression management with graphs: effects on choices. J Inf Syst 16:183-202

Azen R, Budescu DV (2003) Dominance analysis: a method for comparing predictors in multiple regression. Psychol Methods 8:129-148

Becker SW, Brownson FO (1964) What price ambiguity? On the role of ambiguity in decision making. J Political Econ 72:62-73

Borsuk ME, Tomassini L (2005) Uncertainty, imprecision and the precautionary principle in climate change assessment. Water Sci Technol 52:213-225

Brock WA, Xepapadeas A (2003) Regulating nonlinear environmental systems under Knightian uncertainty. In: Stiglitz Festschrift, pp 127-144. MIT Press, Massachusetts

Brown C (2011) Decision-scaling for robust planning and policy under climate uncertainty. World Resources Report Uncertainty Series, Washington, DC

Budescu DV, Fischer I (2001) The same but different: an empirical examination of the reducibility principle. J Behav Decis Mak 14:187-206

Budescu DV, Rantilla AK (2000) Confidence in aggregation of expert opinions. Acta Psychol 104:371-398

Budescu DV, Wallsten TS (1987) Subjective estimation based on precise and vague uncertainties. In: Wright G, Ayton P (eds) Judgmental forecasting. Wiley, NY, pp 63-92

Budescu DV, Wallsten TS (1995) Processing linguistic probabilities: general principles and empirical evidence. In: Busemeyer J, Hastie R, Medin DL (eds) Decision making from a cognitive perspective. Academic Press, San Diego, pp 275-318

Budescu DV, Weinberg S, Wallsten TS (1988) Decisions based on numerically and verbally expressed uncertainties. J Exp Psychol Hum Percept Perform 14:281-294

Budescu DV, Kuhn KM, Kramer KM, Johnson T (2002) Modeling certainty equivalents for imprecise gambles. Organ Behav Hum Decis Process 88:748-768

Camerer C, Weber M (1992) Recent developments in modeling preferences: uncertainty and ambiguity. J Risk Uncertain 5:325-370

Casey JT, Scholz JT (1991) Boundary effects of vague risk information on taxpayer decisions. Organ Behav Hum Decis Process 50:360-394

Casman EAM, Granger M, Dowlatabadi H (1999) Mixed levels of uncertainty in complex policy models. Risk Anal 19:33-42

Chen Z, Epstein LG (2002) Ambiguity, risk and asset returns in continuous time. Econometrica 70:1403-1443

Curley SP, Yates JF (1985) The center and range of the probability interval as factors affecting ambiguity preferences. Organ Behav Hum Decis Process 36:273-287

Curley SP, Yates JF, Abrams RA (1986) Psychological sources of ambiguity avoidance. Organ Behav Hum Decis Process 38:230-256

Dessai S, Hulme M (2007) Assessing the robustness of adaptation decisions to climate change uncertainties: a case study on water resources management in the East of England. Glob Environ Change 17:59-72

Du N, Budescu DV (2005) The effects of imprecise probabilities and outcomes in evaluating investment options. Manage Sci 51:1791-1803

Du N, Budescu DV, Sheely M, Omer TC (2011) The appeal of vague financial forecasts. Organ Behav Hum Decis Process 114:179-189

Ellsberg D (1961) Risk, ambiguity, and the Savage axioms. Q J Econ 75:643-669

Etner J, Jeleva M, Talon JM (2012) Decision theory under ambiguity. J Econ Surv 26:234-260

Fiedler K, Unkelbach C (2011) Lottery attractiveness and presentation mode of probability and value information. J Behav Decis Mak 99:99-115

Fox CR, Rottensterich Y (2003) Partitioning priming in judgments under uncertainty. Psychol Sci 14:195-200

Fox CR, Tversky A (1995) Ambiguity aversion and comparative ignorance. Q J Econ 110:879-895 
Fox CR, Weber M (2002) Ambiguity aversion, comparative ignorance, and decision context. Organ Behav Hum Decis Process 88:476-498

French S (1986) Decision theory: an introduction to the mathematics of rationality. Ellis Horwood Limited, Chichester

Froyn CB (2005) Decision criteria, scientific uncertainty, and the global warming controversy. Mitig Adapt Strateg Glob Change 10:183-211

Gigerenzer G (1994) Why the distinction between single-event probabilities and frequencies is relevant for psychology and vice versa. In: Wright G, Ayton P (eds) Subjective probability. Wiley, New York, pp 129-162

Giles J (2002) When doubt is a sure thing. Nature 418:476-478

Groves DG, Knopman D, Lempert RJ, Berry S, Wainfan L (2007) Presenting uncertainty about climate change to water resource managers, RAND TR-505-NSF. Rand Corporation, Santa Monica

Hall JM, Lempert R, Keller K, Hackbarth A, Mijere C, McInerney D (2012) Robust climate policies under uncertainty: a comparison of info-gap and RDM methods. Risk Anal 32(10):1657-1672

Hey JD, Lotito G, Maffioletti A (2010) The descriptive and predictive adequacy of theories of decision making under uncertainty/ambiguity. J Risk Uncertain 41:81-111

Hof AF, van Vuuren DP, den Elzen M (2010) A quantitative minimax regret approach to climate change: does discounting still matter? Ecol Econ 70:43-51

Hoffrage U, Lindsey S, Hertwig R, Gigerenzer G (2000) Communicating statistical information. Science 290:2261-2262

Hogarth RM, Kunreuther H (1989) Risk, ambiguity and insurance. J Risk Uncertain 2:5-35

Hung S-Y, Ku Y-C, Liang T-P, Lee C-J (2007) Regret avoidance as a measure of DSS success: an exploratory study. Decis Support Syst 42:2093-2106

Ibrekk H, Morgan MG (1987) Graphical communication of uncertain quantities to nontechnical people. Risk Anal 7:519-529

Johnson EJ, Payne JW, Bettman JR (1988) Information displays and preference reversals. Organ Behav Hum Decis Process 42:1-21

Kann A, Weyant JP (2000) Approaches for performing uncertainty analysis in large scale energy/ economic policy models. Environ Model Assess 5:29-46

Keller R (1985a) The effects of problem representation on the sure-thing and substitution principles. Manage Sci 31:738-751

Keller R (1985b) Testing of the 'reduction of compound alternatives' principle. OMEGA Int J Manage Sci 13:349-358

Keller K, Schlesinger M, Yohe G (2008) Managing the risks of climate thresholds: uncertainties and information needs. Clim Change 91:5-10

Keren G, Gerritsen LEM (1999) On the robustness and possible accounts of ambiguity aversion. Acta Psychol 103:149-172

Kleinmuntz D, Schkade D (1993) Information displays and decision processes. Psychol Sci 4:221-227

Kramer KM, Budescu DV (2005) Exploring Ellsberg's paradox in vague-vague cases. In: Zwick R, Rapoport A (eds) Experimental business research, vol III. Kluwer Academic Publishers, Norwell, pp 131-154

Kuhn KM, Budescu DV (1996) The relative importance of probabilities, outcomes, and vagueness in hazard risk decisions. Organ Behav Hum Decis Process 68:301-317

Kuhn KM, Budescu DV, Hershey JR, Kramer KM, Rantilla AK (1999) Attribute tradeoffs in low probability/high consequence risks: the joint effects of dimension preference and vagueness. Risk Decis Policy 4:31-46

Kunreuther H, Meszaros J, Hogarth RM, Spranca M (1995) Ambiguity and underwriter decision processes. J Econ Behav Organ 26:337-352

Kunreuther H, Heal G, Allen M, Edenhofer O, Field CB, Yohee G (2013) Risk management and climate change. Nat Climate Change 3:447-450

Lange A, Treich N (2008) Uncertainty, learning and ambiguity in economic models on climate policy: some classical results and new directions. Clim Change 89:7-21

Lempert RJ, Collins M (2007) Managing the risk of uncertain threshold responses: comparison of robust, optimum, and precautionary approaches. Risk Anal 27:1009-1026

Lempert RJ, Schlesinger ME, Bankes SC (1996) When we don't know the costs or the benefits: adaptive strategies for abating climate change. Clim Change 33:235-274

Lempert RJ, Popper SW, Bankes SC (2003) Shaping the next one hundred years: new methods for quantitative, long-term policy analysis, RAND MR-1626-RPC. RAND, Santa Monica 
Lempert RJ, Nakicenovic N, Sarewitz D, Schlesinger M (2004) Characterizing climate-change uncertainties for decision-makers. Clim Change 65:1-9

Lipkus IM (2007) Numerical, verbal and visual formats of conveying health risks. Suggested best practices and future recommendations. Med Decis Mak 27:696-713

Liverman D, Raven P (eds) (2010) Informing an effective response to climate change. National Academy Press, Washington, DC. http://www.nap.edu/catalog.php?record_id=12784

Loomes G, Pinto-Prades JL, Abellan-Perpinan JM, Rodriguez-Miguez E (2010) Modelling noise and imprecision in individual decisions, Working Papers 10.03, Universidad Pablo de Olavide, Department of Economics. Accessed June 20, 2010 from http://ideas.repec.org/p/pab/wpaper/10.03.html

Loulou R, Kanudia A (1999) Minimax regret strategies for greenhouse gas abatement: methodology and application. Oper Res Lett 25:219-230

Luce RD, Raiffa H (1954) Games and decisions: introduction and critical survey. Dover Publications, New York

Macgregor D, Slovic P (1986) Graphic representation of judgmental information. Hum Comput Interact 2:179-200

Manski CF (2010) Choosing treatment policies under ambiguity. Annu Rev Econ 3:25-49

McInerney D, Lempert R, Keller K (2012) What are robust strategies in the face of uncertain climate threshold responses? Clim Change 112:547-568

Morgan MG, Keith DV (1995) Climate-change-subjective judgments by climate experts. Environ Sci Technol 29:A468-A476

Moss RH, Schneider SH (2000) Uncertainties in the IPCC TAR: recommendations to lead authors for more consistent assessment and reporting. In: Pachuani R, Tanaka K, Taniguchi T (eds) Guidance Papers on the Cross Cutting Issues of the Third Assessment Report of the IPCC, Intergovernmental Panel on Climate Change. World Meteorological Organization, Geneva, pp 33-51

Nordhaus WD (1994) Managing the global commons: the economics of climate change. The MIT press, Cambridge

Onay S, La-ornual D, Öncüler A (2012) The effects of temporal distance on attitudes towards imprecise probabilities and imprecise outcomes. J Behav Decis Mak. doi:10.1002/bdm.1763

Payne JW, Bettman JR, Johnson EJ (1993) The adaptive decision maker. Cambridge University Press, Cambridge

Por H, Budescu DV (2013) Revisiting the gain-loss separability assumption in prospect theory. J Behav Decis Mak. doi:10.1002/bdm.1765

Reeder T, Ranger N (2011) How do you adapt in an uncertain world? Lessons from the Thames Estuary 2100 Project. World Resources Report, Washington, DC

Salvadori L, Van Swol LM, Sniezek JA (2001) Information sampling and confidence within groups and judge advisor systems. Commun Res 28:737-771

Sanfey A, Hastie R (1998) Does evidence presentation format affect judgment? An experimental evaluation of displays of data for judgments. Psychol Sci 9:99-103

Seale D, Rapoport A, Budescu DV (1995) Decision making under strict uncertainty: an experimental test of competitive criteria. Organ Behav Hum Decis Process 64:65-75

Shafir E (1995) Compatibility in cognition and decision. In: Busemeyer JR, Hastie R, Medin DL (eds) Decision making from the perspective of cognitive psychology (The psychology of learning and motivation, vol 32). Academic Press, New York, pp 247-274

Todd PA, Benbasat I (1994) The influence of decision aids on choice strategies under conditions of high cognitive load. IEEE Trans Syst Man Cybern 24:537-547

Trautmann ST, Vieider FM, Wakker PP (2011) Preference reversals for ambiguity aversion. Manage Sci 57:1320-1333

Troffaes MCM (2007) Decision making under uncertainty using imprecise probabilities. Int J Approx Reason 45:17-29

Van den Bergh J (2004) Optimal climate policy is a utopia: from quantitative to qualitative cost-benefit analysis. Ecol Econ 48:385-393

Webster M (2003) Communicating climate change uncertainty to policy-makers and the public-an editorial comment. Clim Change 61:1-8

Welsch H (1995) Greenhouse-gas abatement under ambiguity. Energy Econ 17:91-100

Wilby RL, Dessai S (2010) Robust adaptation to climate change. Weather 65:180-185

Zickfeld K, Levermann A, Morgan MG, Kuhlbrodt T, Rahmstorf S, Keith DW (2007) Expert judgments on the response of the Atlantic meridional overturning circulation to climate change. Clim Change $82: 235-265$ 